Dung Q. Ly, Leonid Paramonov, Calvin Davidson, Jeremy Ramsden, Helen Wright, Nick Holliman, Jerry Hagon, Malcolm Heggie and Charalampos Makatsoris

\title{
The Matter Compiler-towards atomically precise engineering and manufacture
}

ABSTRACT. The Matter Compiler is a novel framework for a computer toolset that allows the design of arbitrary molecular structures and their assembly by means of radical synthesis pathways that resemble "pick and place". Such synthesis pathways have been demonstrated experimentally in the literature in the recent past using scanning probe microscopes (SPM). The Matter Compiler framework entails first-principles modelling and novel control approaches that together assess the feasibility of user-defined molecular structures, screen alternative options for their assembly and finally suggest synthesis by means of physical equipment such as scanning probe microscopes. Nanoscale structure design is driven by first-principles calculations as well as by any relevant physical and chemical knowledge that can be accessed by the system from chemical databases. The first main consideration is therefore a suitable modelling methodology enabling the design and assessment of user-defined molecular structures. This entails the computer representation of such structures, methods to access and use available chemical and physical knowledge and, subsequently, an overall approach to plan the synthesis of such structures. Currently, it is assumed that assembly takes place on suitable substrates molecule-bymolecule and uses first-principles calculations to determine synthesis pathways (plans) on those substrates. The second main consideration is to use those synthesis plans to determine how synthesis will take place within a physical instrument. For scanning probes, for example, this translates to a collection of set points for the instrument controller to follow that collectively comprise the trajectory of the scanning probe for each step of the process. Image processing for monitoring progress, visualization and haptic interaction are also part of the Matter Compilerthey provide insight, feedback and determination of intervention strategies in case of errors. In this paper a description of the Matter Compiler methodology and framework architecture is presented. Some computational results are also presented.

Nanotechnology Perceptions 7 (2011) 199-217

Nonsubscribers: purchase individual article 